\title{
ON REPRESENTATIONS OF LIE ALGEBRAS OF A GENERALIZED TAVIS-CUMMINGS MODEL
}

\author{
L. A. M. HANNA
}

Received 13 February 2002 and in revised form 9 July 2002

Consider the Lie algebras $L_{r, t}^{s}:\left[K_{1}, K_{2}\right]=s K_{3},\left[K_{3}, K_{1}\right]=r K_{1},\left[K_{3}, K_{2}\right]=$ $-r K_{2},\left[K_{3}, K_{4}\right]=0,\left[K_{4}, K_{1}\right]=-t K_{1}$, and $\left[K_{4}, K_{2}\right]=t K_{2}$, subject to the physical conditions, $K_{3}$ and $K_{4}$ are real diagonal operators representing energy, $K_{2}=K_{1}^{\dagger}$, and the Hamiltonian $H=\omega_{1} K_{3}+\left(\omega_{1}+\omega_{2}\right) K_{4}+$ $\lambda(t)\left(K_{1} e^{-i \phi}+K_{2} e^{i \phi}\right)$ is a Hermitian operator. Matrix representations are discussed and faithful representations of least degree for $L_{r, t}^{s}$ satisfying the physical requirements are given for appropriate values of $r, s, t \in \mathbb{R}$.

\section{Introduction}

Introducing an algebraic method to solve certain types of linear partial differential equations, Steinberg [6] exploited the Lie-algebraic decomposition formulas of Baker, Campbell, Hausdorff, and Zassenhaus (cf. [7]) and their matrix realization. A faithful matrix representation of low degree is required. In $[2,3,4]$, the faithful matrix representations of least degree were discussed for the Lie algebra $L_{r}^{s}$ generated by $K_{+}$, $K_{-}$, and $K_{0}$ satisfying the commutation relations: $\left[K_{0}, K_{ \pm}\right]= \pm r K_{ \pm}$and $\left[K_{+}, K_{-}\right]=s K_{0}$ subject to the physical properties $K_{-}=K_{+}^{\dagger}$ († for Hermitian conjugation), $K_{0}$ is a real diagonal operator, and $\left(K_{+}+K_{-}\right)$is real. The Lie algebra $L_{r}^{s}$ was introduced as a generalization of the coupled quantized harmonic oscillators [5] namely, the model of light amplifier $L_{1}^{-2}$, and the model of two-level optical atom $L_{1}^{2}$, whose Hamiltonian model $H=K_{0}+\lambda\left(K_{+}+K_{-}\right), \lambda$ is the coupling parameter. Note that, $L_{2}^{1}$ is exactly the Lie algebra $\mathfrak{s l}(2)$.

In this paper, $L_{r, t}^{s}$ is considered to be the Lie algebra generated by $K_{1}$, $K_{2}, K_{3}$, and $K_{4}$, satisfying the commutation relations: $\left[K_{1}, K_{2}\right]=s K_{3}$, 
$\left[K_{3}, K_{1}\right]=r K_{1},\left[K_{3}, K_{2}\right]=-r K_{2},\left[K_{3}, K_{4}\right]=0,\left[K_{4}, K_{1}\right]=-t K_{1},\left[K_{4}, K_{2}\right]=$ $t K_{2}$, subject to the physical conditions, $K_{3}$ and $K_{4}$ are real diagonal operators representing energy, $K_{2}=K_{1}^{\dagger}$, and the Hamiltonian $H=\omega_{1} K_{3}+$ $\left(\omega_{1}+\omega_{2}\right) K_{4}+\lambda(t)\left(K_{1} e^{-i \phi}+K_{2} e^{i \phi}\right)$ is a Hermitian operator. The Lie algebra $L_{r, t}^{s}$ is introduced as a generalization of the Tavis-Cummings model namely, $L_{2,1}^{1}$ in [1]. Obviously, the subalgebra of $L_{r, t}^{s}$ generated by $K_{1}$, $K_{2}$, and $K_{3}$ in respective with $K_{+}, K_{-}$, and $K_{0}$ is a generalization of $L_{r}^{s}$, when dropping the physical condition $\left(K_{+}+K_{-}\right)$must be real. That condition forced the representation matrices of $K_{+}$and $K_{-}$to be real, $[2,3,4]$. Faithful matrix representations of least degree are discussed for $L_{r, t}^{s}$ for appropriate values of $r, s, t \in \mathbb{R}$.

Unless otherwise stated, $I_{m}$ is the identity matrix of degree $m, O$ is the zero matrix of appropriate size, $\mathbb{N}=\{1,2, \ldots, n\}$ and $A=\left[a_{i j}\right], B=\left[b_{i j}\right]$, $C=\left[\delta_{i j} c_{i j}\right]$, and $D=\left[\delta_{i j} d_{i j}\right]$ are $n \times n$ real matrices, where the matrices $X=A+i B, Y=A^{T}-i B^{T}, C$, and $D$ are representation matrices for $K_{1}$, $K_{2}, K_{3}$, and $K_{4}$, respectively; $i=\sqrt{-1}$. All representations for $L_{r, t}^{s}$ under consideration are supposed to satisfy the above-mentioned physical requirements.

LEMMA 1.1. The Lie algebra $L_{r, t}^{s}$ can be defined by

$$
\left[K_{1}, K_{2}\right]=s K_{3}, \quad\left[K_{3}, K_{1}\right]=r K_{1}, \quad\left[K_{4}, K_{1}\right]=-t K_{1},
$$

where $K_{3}$ and $K_{4}$ are real diagonal operators and $K_{2}=K_{1}^{\dagger}$.

Proof. Indeed $-r K_{2}=-\left(r K_{1}\right)^{\dagger}=-\left[K_{3}, K_{1}\right]^{\dagger}=\left[K_{3}, K_{2}\right]$ and similarly, for the relation $\left[K_{4}, K_{2}\right]=t K_{2}$. Since $K_{3}$ and $K_{4}$ are diagonal, they commute. The Hermiticity of the Hamiltonian follows since $\omega_{1}, \omega_{2}, \lambda(t) \in \mathbb{R}$.

As a necessity of Lemma 1.1 we have the following lemma.

Lemma 1.2. The matrices $A, B, C$, and $D$ satisfy the following:

(i) $\left[A, B^{T}\right]$ is a symmetric matrix,

(ii) $\left[A, A^{T}\right]+\left[B, B^{T}\right]=s C$,

(iii) $[C, A]=r A,[C, B]=r B$,

(iv) $[D, A]=-t A,[D, B]=-t B$.

LeMmA 1.3. Let $L, M$, and $K$ be $n \times n$ matrices such that $[L, M]=a K, a \neq 0$, then $\operatorname{trace}(K)=0$.

LEMMA 1.4. Let $p, q \in \mathbb{N}$, and $\sigma=(p q)$ be a transposition. The representation obtained by applying $\sigma$ to the rows as well as to the columns of $X, Y, C$, and $D$ is a conjugate representation for $L_{r, t}^{s}$ and satisfies the physical requirements. 
Proof. Let $P$ be the elementary matrix obtained by applying $\sigma$ to the rows of $I_{n}$. Since $P=P^{-1}=P^{T}=P^{\dagger}$, then the proof of the lemma follows.

Since $[C, X]=r X$, then for all $i, j \in \mathbb{N}$ we have,

$$
a_{i j}\left(c_{i i}-c_{j j}-r\right)=0, \quad b_{i j}\left(c_{i i}-c_{j j}-r\right)=0 .
$$

Similarly, from Lemma 1.2(iv),

$$
a_{i j}\left(d_{i i}-d_{j j}+t\right)=0, \quad b_{i j}\left(d_{i i}-d_{j j}+t\right)=0 .
$$

If $x_{i j} \neq 0$, then from (1.2) and (1.3)

$$
c_{i i}-c_{j j}=r, \quad d_{j j}-d_{i i}=t .
$$

Since $[X, Y]=s C$, then for each $i \in \mathbb{N}$ we have,

$$
s c_{i i}=\sum_{l=1}^{n}\left(\left|x_{i l}\right|^{2}-\left|x_{l i}\right|^{2}\right)=\sum_{l=1}^{n}\left(a_{i l}^{2}-a_{l i}^{2}+b_{i l}^{2}-b_{l i}^{2}\right) .
$$

LEMMA 1.5. If $t^{2}+r^{2} \neq 0$, then

(1) $x_{i i}=0$, for all $i \in \mathbb{N}$,

(2) if $x_{i j} \neq 0$ then $x_{j i}=0$, for all $i, j \in \mathbb{N}$.

Proof. If $r \neq 0$, then from (1.2) we have, for each $i \in \mathbb{N}$, that $x_{i i}=0$. Also, if $x_{i j} \neq 0$, then $c_{j j}-c_{i i}-r=-2 r$, thus $x_{j i}=0$. Similarly, when $t \neq 0$.

LEMMA 1.6. If $s \neq 0$, then

(1) $\operatorname{trace}(C)=0$,

(2) if $x_{i j} \neq 0$ then, for $i, j \in \mathbb{N}$

$$
r=\frac{1}{S}\left[\sum_{l=1}^{n}\left(\left|x_{i l}\right|^{2}-\left|x_{l i}\right|^{2}-\left|x_{j l}\right|^{2}+\left|x_{l j}\right|^{2}\right)\right] .
$$

Proof. Since $[X, Y]=s C$ then from Lemma 1.3 , trace $(C)=0$. The proof of (2), follows from (1.4) and (1.5).

We build the representation matrices starting with $C$.

Remark 1.7. Using Lemma 1.4, $C$ can be rearranged into $k$ diagonal blocks, the $i$ th diagonal block consists of the $k_{i}$ scalar matrices, $\left\{c_{i} I_{m_{i, 0}},\left(c_{i}-\right.\right.$ $\left.r) I_{m_{i, 1}}, \ldots,\left[c_{i}-r\left(k_{i}-1\right)\right] I_{m_{i,\left(k_{i}-1\right)}}\right\}$, where $m_{i, j}$ is the repetitions of $\left(c_{i}-r j\right)$ 
in the diagonal of $C$; for $i=1,2, \ldots, k$ and $j=0,1, \ldots, k_{i}-1$. Thus,

$$
\begin{aligned}
C=\operatorname{diag}\{ & c_{1} I_{m_{1,0}},\left(c_{1}-r\right) I_{m_{1,1},} \ldots,\left[c_{1}-r\left(k_{1}-1\right)\right] I_{m_{1,\left(k_{1}-1\right)}}, \ldots, \\
& c_{i} I_{m_{i, 0}}\left(c_{i}-r\right) I_{m_{i, 1}}, \ldots,\left[c_{i}-r\left(k_{i}-1\right)\right] I_{m_{i,\left(k_{i}-1\right)}, \ldots,}, \\
& \left.c_{k} I_{m_{k, 0}}\left(c_{k}-r\right) I_{m_{k, 1}}, \ldots,\left[c_{k}-r\left(k_{k}-1\right)\right] I_{m_{k,\left(k_{k}-1\right)}}\right\},
\end{aligned}
$$

where

$$
\begin{gathered}
c_{i} \neq c_{j}, \quad \text { whenever } i \neq j, \quad \text { for } i, j=1,2, \ldots, k, \\
{\left[c_{i}-r j\right]-c_{i+1} \neq r, \quad \text { for } j=0, \ldots, k_{i}-1 ; i=1,2, \ldots, k-1 .}
\end{gathered}
$$

The $i$ th diagonal block of $C$ is called the $c_{i}$-block and $k_{i}$ is its length. Any diagonal entry $c$ of $C$ such that $c=c_{i}-r l$, for $l \geq 0$ then $0 \leq l \leq k_{i}-$ 1 for some $i=1, \ldots, k$, that is, $c$ belongs to the $c_{i}$-block. If $c_{i}-l_{1} r=c_{j}-$ $l_{2} r, 0 \leq l_{1} \leq k_{i}-1,0 \leq l_{2} \leq k_{j}-1$, then $c_{i}$ and $c_{j}$ are in the same block, violating (1.9).

We use the notations given in Remark 1.7.

\section{Faithful representations for $L_{r, t}^{s}$ where $r s \neq 0$}

LemMA 2.1. The matrices $A$ and $B$ can be partitioned into submatrices of the same size corresponding to those of $C$. The nonzero submatrices of $A$ and $B$ are all off-diagonal submatrices.

Proof. From (1.2), the diagonal submatrices of $A$ and $B$ are square zero submatrices of orders $m_{1,0}, \ldots, m_{k,\left(k_{k}-1\right)}$, in respective to those of $C$. Let $c_{i i}, c_{j j}$, and $c_{l l} ; i, j, l \in \mathbb{N}$, be from different diagonal submatrices of $C$, and suppose that $a_{i j} \neq 0$ and $a_{i l} \neq 0$, then from (1.2), $c_{l l}=c_{j j}$ contradicting (1.8). Similarly, if $a_{j i}$ and $a_{l i}$ are from different submatrices in $A$ they cannot be both nonzero. In view of (1.2), only the off-diagonal submatrices of $A$ may be nonzero. Thus we have, $A=\left[A_{i j}\right]$ where $A_{i j}=O$, for $j \neq i+1$. And similarly for $B$.

LEMMA 2.2. For $k>1$, if $k_{i}=1$, for some $i=1,2, \ldots, k$, then $L_{r, t}^{s}$ has a representation of degree $n-m_{i, 0}$. Moreover, if the entries in the ith row and the ith column of $X$ are all zeros, then $L_{r, t}^{s}$ has a representation of degree $n-1$.

Proof. We use Lemma 1.4 so that the $c_{i}$-block becomes the first block of the main diagonal of $C$. Since for all $j \in \mathbb{N}, 1 \leq i \leq m_{1,0},\left|c_{i i}-c_{j j}\right| \neq r$, otherwise $k_{i}>1$, then from (1.2) the representation is fully reducible since, $A=\left[\begin{array}{cc}0 & 0 \\ 0 & A^{\prime}\end{array}\right], B=\left[\begin{array}{cc}0 & 0 \\ 0 & B^{\prime}\end{array}\right], C=\left[\begin{array}{cc}C_{1}^{\prime} & 0 \\ 0 & C_{2}^{\prime}\end{array}\right]$, and $D=\left[\begin{array}{cc}D_{1}^{\prime} & 0 \\ 0 & D_{2}^{\prime}\end{array}\right]$. The matrices 
$X^{\prime}=A^{\prime}+i B^{\prime}, Y^{\prime}=X^{\prime \dagger}, C_{2}^{\prime}$, and $D_{2}^{\prime}$ are all of degree $n-m_{i, 0}$ and satisfy the lemma. Similar argument holds when the entries in the $i$ th row and the $i$ th column of $X$ are all zeros.

So, it can be assumed that if $k>1$ then $k_{i}>1 ; i=1, \ldots, k$. And for $X \neq$ $O$, if the entries of the $i$ th row of $X$ are all zeros, then those of the $i$ th column are not all zeros, and vice versa, in such cases, we get from (1.5) that $s c_{i i} \neq 0$.

TheOREM 2.3. If $r s<0$, then $X=Y=C=O$.

Proof. If $k=1$ and $k_{1}=1$, then from (1.2) $X=Y=O$. If $X=O$, then from (1.5) $C=O$. Suppose that $X \neq O$, there are only two cases to consider namely, the case where $k=1$ and $k_{1}>1$, and the case where $k>1$. In both cases $k_{1}>1$, from Lemma 2.1 the first $m_{1,0}$ columns of $X$ are zero columns, and from Lemma 2.2 there must be an $x_{1, j} \neq 0$ for some $m_{1,0}<$ $j \leq\left(m_{1,0}+m_{1,1}\right)$. Thus from (1.5),

$$
s c_{11}=s c_{1}=\sum_{l=1}^{n}\left(\left|x_{1 l}\right|^{2}-0\right)>0 \text {. }
$$

Let $\alpha=m_{1,0}+m_{1,1}+\cdots+m_{1,\left(k_{1}-2\right)}$. If $k>1$, we get from (1.9), $\left[c_{1}-r\left(k_{1}-\right.\right.$ $1)]-c_{2} \neq r$, thus from (1.2), the rows $\alpha+1, \alpha+2, \ldots, \alpha+m_{1,\left(k_{1}-1\right)}$ are zero rows of $X$. If $k=1$ and $k_{1}>1$, we get from Lemma 2.1 that the mentioned rows are zero rows of $X$, being the last rows of $X$. In both cases, from Lemma 2.2 there must be an $x_{i, \alpha+1} \neq 0$ for some $\left[\alpha-m_{1,\left(k_{1}-2\right)}\right]<i \leq \alpha$. From (1.5),

$$
s c_{\alpha+1, \alpha+1}=s\left[c_{1}-r\left(k_{1}-1\right)\right]=\sum_{l=1}^{n}\left(0-\left|x_{l, \alpha+1}\right|^{2}\right)<0 .
$$

If $s>0$, then $c_{1}>0$ by $(2.1)$, since $r<0$, then $\left[c_{1}-r\left(k_{1}-1\right)\right]>0$, violating (2.2). Similarly, if $s<0$, we get from (2.1), $\left[c_{1}-r\left(k_{1}-1\right)\right]<0$, violating (2.2).

We conclude this section by introducing the $2 \times 2$ representation matrices $X, Y, C$, and $D$ of $K_{1}, K_{2}, K_{3}$, and $K_{4}$, respectively, for $r s>0, t \in \mathbb{R}$

$$
\begin{aligned}
& X=\left[\begin{array}{cc}
0 & a \pm i \sqrt{r s / 2-a^{2}} \\
0 & 0
\end{array}\right], \quad Y=\left[\begin{array}{cc}
0 & 0 \\
a \mp i \sqrt{r s / 2-a^{2}} & 0
\end{array}\right], \\
& C=\left[\begin{array}{cc}
r / 2 & 0 \\
0 & -r / 2
\end{array}\right], \quad D=\left[\begin{array}{cc}
b & 0 \\
0 & b+t
\end{array}\right],
\end{aligned}
$$


for any $a, b \in \mathbb{R}$ such that $|a| \leq \sqrt{r s / 2}$ and for the linear independency of $C$ and $D$, take $b \neq-t / 2$. These representations are faithful. The $2 \times 2$ representation matrices $X, Y, C$, and $D$ generalize those given in [1].

Clearly, the vector space spanned by $X, Y$, and $C$ is $\mathfrak{s l}(2, \mathbb{C})$, as a vector space. The representation matrices of $L_{r}^{s}$, in [2], are for the special cases, $a^{2}=r s / 2$.

\section{Faithful representations for $L_{r, t}^{s}$ where $r s t=0$}

The case where $r s \neq 0$ and $t=0$ was considered in the previous section. So, if $s \neq 0$ we only need to consider the case where $r=0$ and $t$ is any real number.

\subsection{For $s \neq 0, r=0$, and $t \in \mathbb{R}$}

Since $r=0$ then any $c_{i}$-block of the matrix $C$ has length $k_{i}=1$. So, we have $C=\operatorname{diag}\left(c_{1} I_{m_{1}}, \ldots, c_{k} I_{m_{k}}\right)$ where $c_{i} \neq c_{j}$ whenever $i \neq j ; i, j=1, \ldots, k$.

Remark 3.1. If $X$ commutes with $Y=X^{\dagger}$, then $X$ is a normal matrix, and there exists a unitary matrix $U$ such that $X=U^{\dagger} Z U$ for some complex diagonal matrix $Z$. If $U$ commutes with $C$ and $D$, then the diagonal matrices $Z, \bar{Z}, C$, and $D$ are representation matrices for $K_{1}, K_{2}, K_{3}$, and $K_{4}$, respectively, and satisfy the physical requirements. We take $U=I_{n}$ when $X$ is diagonal.

Lemma 3.2. If $C=\operatorname{diag}\left(c_{1} I_{m_{1}}, \ldots, c_{k} I_{m_{k}}\right)$ for different $c_{i}$ 's, then the representation is fully reducible into representations of degrees $m_{1}, \ldots, m_{k}$.

Proof. The matrix $D$ is diagonal and from (1.2), $x_{i j}=x_{j i}=y_{i j}=y_{j i}=0$, whenever $c_{i i} \neq c_{j j} ; i, j \in \mathbb{N}$.

Lemma 3.3. Let $K=\left[K_{i j}\right]$ be a partitioned matrix which is normal whose diagonal blocks are $k$ square matrices. If $K_{i j}=O$ whenever $j \neq i+1($ or $j \neq i-1)$; $i, j=1, \ldots, k$. Then $K=O$.

Proof. Let $K=\left[k_{i j}\right]$ be an $n \times n$ matrix, then for each $i \in \mathbb{N}$,

$$
\sum_{l=1}^{n}\left|k_{i l}\right|^{2}=\sum_{l=1}^{n}\left|k_{l i}\right|^{2}
$$

Let the diagonal blocks of $K$ be of degrees $i_{1}, \ldots, i_{k}$, respectively. If $K_{i j}=O$ whenever $j \neq i+1 ; i, j=1, \ldots, k$, then the first $i_{1}$ rows of $K$ are zeros, thus from (3.1) the first $i_{1}$ columns of $K$ are zeros. Continuing like that in less 
than $k$ steps, it can be shown that $K=O$. Hence the proof of the lemma follows.

TheOREM 3.4. The matrix $C=O$, in any representation of $L_{0, t}^{s}$. If $s t \neq 0$, then $X=Y=O$.

Proof. Suppose $C \neq O$, we use Lemma 1.4 so that $c_{1} \neq 0$, from (1.5) and Lemma 3.2, $m_{1} s c_{1}=\sum_{i=1}^{m_{1}} s c_{i i}=\sum_{i=1}^{m_{1}} \sum_{l=1}^{m_{1}}\left(\left|x_{i l}\right|^{2}-\left|x_{l i}\right|^{2}\right)=0$, but $m_{1} s c_{1} \neq 0$. Then $C=O$. Thus from Lemma $1.1, X$ is a normal matrix. If $t \neq 0$, we use Lemma 1.4 , so that

$$
\begin{aligned}
D=\operatorname{diag}\{ & d_{1} I_{m_{1,0}^{\prime}}\left(d_{1}+t\right) I_{m_{1,1}^{\prime}}, \ldots,\left[d_{1}+t\left(k_{1}^{\prime}-1\right)\right] I_{m_{1,\left(k_{1}^{\prime}-1\right)}^{\prime}}, \ldots, d_{i} I_{m_{i, 0}^{\prime}}, \\
& \left(d_{i}+t\right) I_{m_{i, 1}^{\prime}}, \ldots,\left[d_{i}+t\left(k_{i}^{\prime}-1\right)\right] I_{m_{i,\left(k_{i}^{\prime}-1\right)}^{\prime}}, \ldots, d_{k^{\prime}} I_{m_{k^{\prime}, 0}^{\prime}} \\
& \left.\left(d_{k^{\prime}}+t\right) I_{m_{k^{\prime}, 1}^{\prime}}, \ldots,\left[d_{k^{\prime}}+t\left(k_{k^{\prime}}^{\prime}-1\right)\right] I_{m_{k^{\prime},\left(k_{k^{\prime}}^{\prime}-1\right)}}\right\}
\end{aligned}
$$

where $m_{i, j}^{\prime}$ is the repetitions of $\left(d_{i}+t j\right)$ in the diagonal of $D$; for $i=1, \ldots, k^{\prime}$ and $j=0, \ldots, k_{i}^{\prime}-1$ such that

$$
\begin{gathered}
d_{i} \neq d_{j}, \quad \text { whenever } i \neq j, \quad \text { for } i, j=1,2, \ldots, k^{\prime}, \\
d_{i+1}-\left[d_{i}+t j\right] \neq t, \quad \text { for } j=0, \ldots, k_{i}^{\prime}-1 ; i=1,2, \ldots, k^{\prime}-1 .
\end{gathered}
$$

From (1.3), X can be partitioned into submatrices of the same sizes corresponding to those of $D$, whose nonzero submatrices are off-diagonal submatrices. Then by Lemma $3.3 X=Y=O$.

If $t=0$ then from Lemma 1.1, the generators commute and such a case can be considered as a special case of $L_{0,0}^{0}$ of Section 3.3, with $C=O$.

\subsection{For $s=0$ and $r^{2}+t^{2} \neq 0$}

From (1.5) as $s=0$, then (3.1) holds. If the $i$ th row (or column) of $X$ consists entirely of zeros, the $i$ th column (or row) also, consists entirely of zeros and both can be omitted by the following lemma whose proof is analogous to that of Lemma 2.2. So, if $X \neq O$, it can be considered that $X$ has no zero row or zero column.

LEMMA 3.5. If $X$ has $m$ zero rows (or columns), where $0 \leq m<n$, then $L_{r, t}^{s}$ has a representation of degree $n-m$. 
Theorem 3.6. If $s=0$ and $r^{2}+t^{2} \neq 0, L_{r, t}^{s}$ has no faithful representations. In any representation, $X=Y=O$.

Proof. If $r \neq 0$, arrange $C$ as in Remark 1.7 otherwise, let $D$ as in the proof of Theorem 3.4. In view of Lemma 1.5, $X$ can be partitioned into submatrices of the same sizes corresponding to those of $C$ when $r \neq 0$ or to those of $D$ otherwise. The nonzero submatrices of $X$ are all off diagonal submatrices. As $s=0$ then $X$ is normal and from Lemma 3.3, we get $X=Y=O$.

\subsection{For $s=r=t=0$}

Although physically is not applicable, but for the sake of completeness, we consider the case when $K_{1}, K_{2}, K_{3}$, and $K_{4}$ are commutant operators.

THEOREM 3.7. The representations of $L_{0,0}^{0}$ are conjugate to representations where $K_{1}, K_{2}, K_{3}$, and $K_{4}$ are represented by diagonal matrices.

Proof. Let $X=U^{\dagger} Z U$ for a unitary matrix $U$ and a complex diagonal matrix $Z$. We claim that $U$ commutes with $C$ and $D$, then the theorem holds by using Remark 3.1. We induce on $n$, the degree of the representation and prove the cases when $X$ is not diagonal.

For $n=2$ : if $X$ is not diagonal then from (1.4), both $C$ and $D$ are scalar matrices and both commute with $U$.

For $n=3$ : if the diagonal elements of $C$ (or $D$ ) are all different, then $X$ must be diagonal. If $X$ has two nonzero elements $x_{i j}$ and $x_{l m}$, from (1.4), both are nondiagonal elements where $x_{l m}$ is not the $x_{j i}$, then $C$ and $D$ are scalar matrices and both commute with $U$. Otherwise, we use Lemma 1.4, so that $X=\left[\begin{array}{ll}X^{\prime} & O \\ O & g\end{array}\right]$, thus from (1.2) and (1.3) $C=\left[\begin{array}{cc}c_{2} I_{2} & O \\ O & a\end{array}\right]$ and $D=$ $\left[\begin{array}{cc}d I_{2} & O \\ O & b\end{array}\right]$, for some $a, b, c, d \in \mathbb{R} ; g \in \mathbb{C}$, where $X^{\prime}$ is not a diagonal matrix. That requires $X^{\prime}$ to be a normal matrix. So, there exists a unitary matrix $U^{\prime}$ such that $X^{\prime}=U^{\prime \dagger} M U^{\prime}$, for some complex diagonal matrix $M$. Obviously, $U^{\prime}$ commutes with $c I_{2}$ and $d I_{2}$. Let $U=\left[\begin{array}{ll}U^{\prime} & O \\ O & 1\end{array}\right]$, and $Z=\operatorname{diag}(M, g)$ then $U$ commutes with $C$ and $D$.

Assume that the theorem is true for $n<m$.

For $n=m$ : if both $C$ and $D$ are scalar matrices, then $U$ commutes with $C$ and $D$. If either $C$ or $D$ is not a scalar matrix, $C$ say, then we use Lemma 1.4 to rearrange $C$ so that $C=\operatorname{diag}\left(c_{1} I_{m_{1}}, \ldots, c_{k} I_{m_{k}}\right)$ for different $c_{i}^{\prime} s$, from (1.2) $X=\operatorname{diag}\left(X_{1}, \ldots, X_{k}\right)$ where $X_{i}$ is a square matrix of order $m_{i}<m$. Also, $D$ can be considered as $D=\operatorname{diag}\left(D_{1}, \ldots, D_{k}\right)$ where $D_{i}$ is a diagonal matrix of degree $m_{i}$. Hence, the representation is fully reducible into representations of degrees $m_{i}, i=1, \ldots, k$. Since $X$ is normal then $X_{i}$ is normal for $i=1, \ldots, k$. Thus there exists a unitary matrix $U_{i}$ such that 
$X_{i}=U_{i}^{\dagger} Z_{i} U_{i}$ for some complex diagonal matrix $Z_{i}, i=1, \ldots, k$. From the induction $U_{i}$ commutes with $c_{i} I_{m_{i}}$ and $D_{i}$. Let $U=\operatorname{diag}\left(U_{1}, \ldots, U_{k}\right)$ and $Z=\operatorname{diag}\left(Z_{1}, \ldots, Z_{k}\right)$, then $U$ commutes with $C$ and $D$.

THEOREM 3.8. The Lie algebra $L_{0,0}^{0}$ has faithful representations of degree 4 as the least degree.

Proof. Any linearly independent diagonal matrices $Z, \bar{Z}, C$, and $D$, of degree 4 , with $C$ and $D$ are real, are representation matrices for $K_{1}, K_{2}$, $K_{3}$, and $K_{4}$, respectively, of a faithful representation.

We conclude the paper by mentioning the cases where $L_{r, t}^{s}$ has faithful matrix representations satisfying the physical requirements.

Summary 3.9. It is assumed that all representations of $L_{r, t}^{s}$ must satisfy the physical requirements.

(1) For $r s>0, t \in \mathbb{R}, L_{r, t}^{s}$ has faithful representations of degree 2 as the least degree.

(2) For $r=s=t=0, L_{0,0}^{0}$ has faithful representation of degree 4 as the least degree where the representation matrices are linearly independent diagonal matrices, with $C$ and $D$ are real matrices.

\section{Acknowledgments}

The author is grateful to Prof. S. S. Hassan, Ain Shams University for suggesting the problem and fruitful discussions and to Prof. S. Singh, King Saud University for reading the manuscript. The support of Kuwait University is also appreciated.

\section{References}

[1] M. A. Bashir and M. S. Abdalla, The most general solution for the wave equation of the transformed Tavis-Cummings model, Phys. Lett. A 204 (1995), no. 1, $21-25$.

[2] L. A. M. Hanna, On the matrix representation of Lie algebras for quantized Hamiltonians and their central extensions, Riv. Mat. Univ. Parma (5) 6 (1997), 511 .

[3] A note on the matrix representations of the Lie algebras $L_{r}^{s}$ for quantized Hamiltonians where rs = 0, Riv. Mat. Univ. Parma (6) 1 (1998), 149-154.

[4] L. A. M. Hanna, M. E. Khalifa, and S. S. Hassan, On representations of Lie algebras for quantized Hamiltonians, Linear Algebra Appl. 266 (1997), 6979.

[5] R. J. C. Spreeuw and J. P. Woerdman, Optical atoms, Prog. Opt. 31 (1993), 263-319. 


\section{On representations of Lie algebras}

[6] S. Steinberg, Applications of the Lie algebraic formulas of Baker, Campbell, Hausdorff, and Zassenhaus to the calculation of explicit solutions of partial differential equations, J. Differential Equations 26 (1977), no. 3, 404-434.

[7] - Lie series, Lie transformations, and their applications, Lie Methods in Optics (León, 1985) (J. S. Mondragón and K. B. Wolf, eds.), Lecture Notes in Phys., vol. 250, Springer, Berlin, 1986, pp. 45-103.

L. A. M. Hanna: Department of Mathematics and Computer Science, Faculty of Science, Kuwait University, P.O. Box 5969, Safat 13060, Kuwait

E-mail address: hannalam@mcs.sci.kuniv.edu.kw 


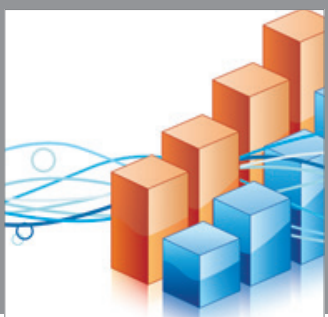

Advances in

Operations Research

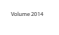

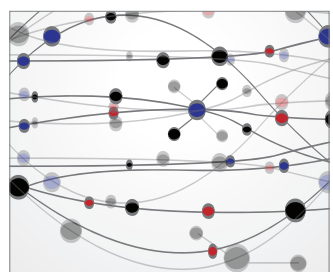

\section{The Scientific} World Journal
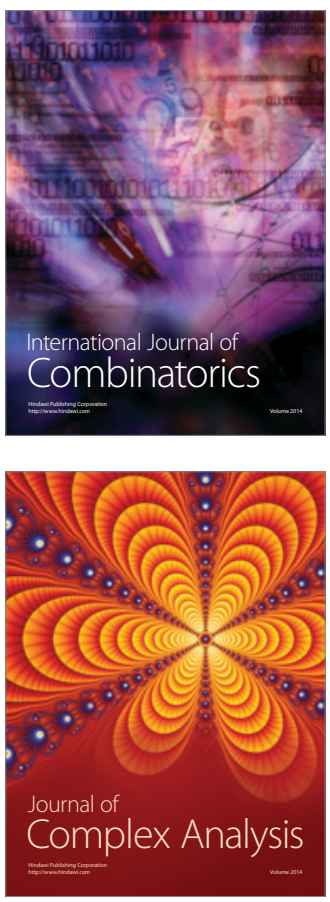

International Journal of

Mathematics and

Mathematical

Sciences
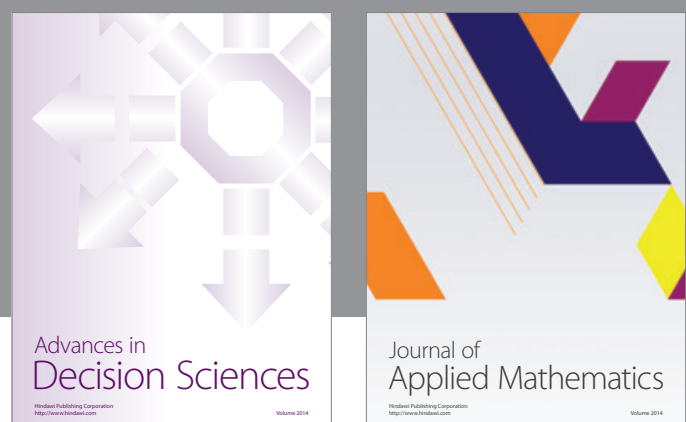

Journal of

Applied Mathematics
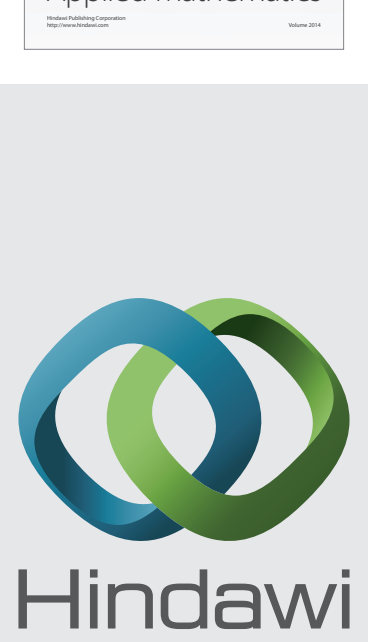

Submit your manuscripts at http://www.hindawi.com
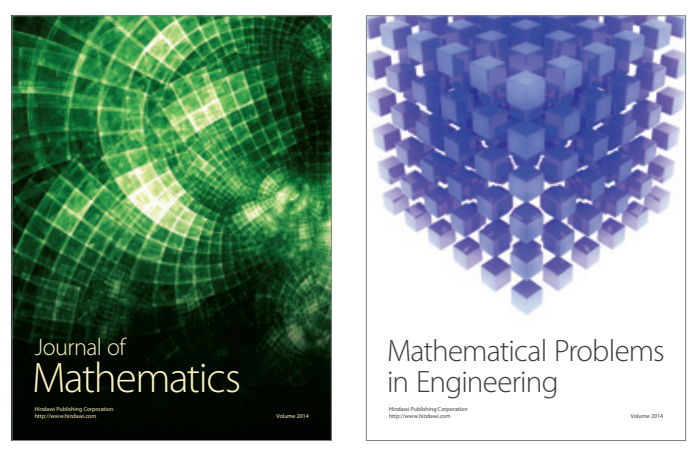

Mathematical Problems in Engineering
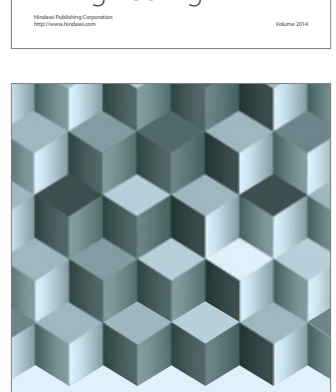

Journal of

Function Spaces
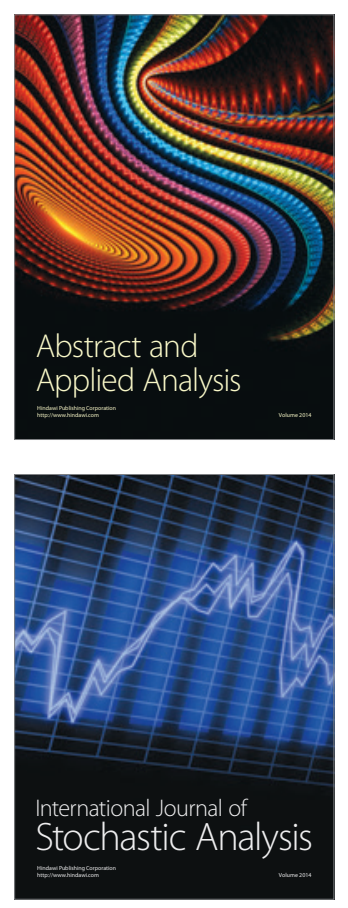

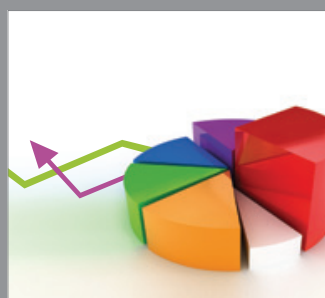

ournal of

Probability and Statistics

Promensencen
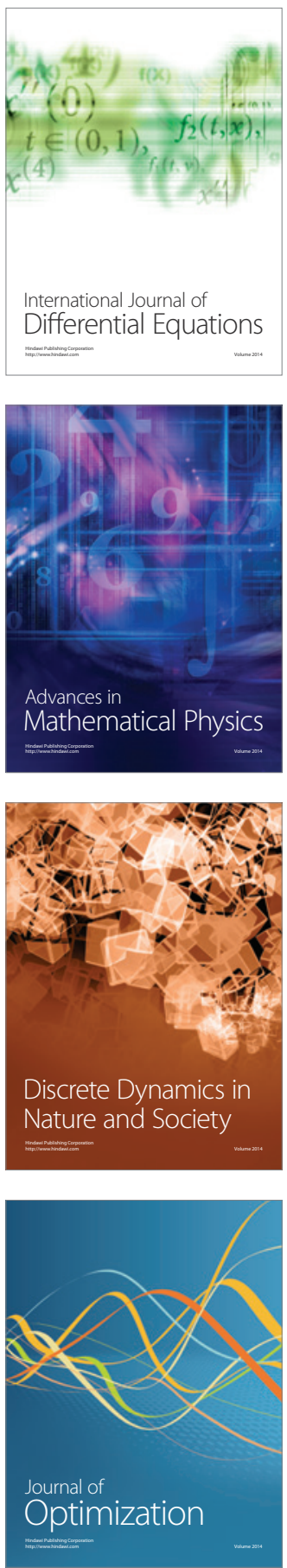